\title{
SIRT6 inhibits proliferation and invasion in osteosarcoma cells by targeting $\mathrm{N}$-cadherin
}

\author{
$\mathrm{YI} \mathrm{GAO}^{1}$, YUXING $\mathrm{QU}^{2}$, QI ZHOU ${ }^{2}$ and $\mathrm{YONG} \mathrm{MA}^{1}$ \\ ${ }^{1}$ Department of Orthopedics and Traumatology, First Clinical Medical College, \\ Nanjing University of Traditional Chinese Medicine, Nanjing, Jiangsu 210023; \\ ${ }^{2}$ Department of Orthopedics, Changzhou City Hospital of Traditional Chinese Medicine, \\ Changzhou, Jiangsu 213000, P.R. China
}

Received May 30, 2017; Accepted August 2, 2018

DOI: $10.3892 / \mathrm{ol} .2018 .9676$

\begin{abstract}
SIRT6, is a member of the NAD-dependent sirtuin family of enzymes, and has been reported as a novel tumor suppressor gene or oncogene, dependent on the type of cancer. However, the role of SIRT6 in osteosarcoma has not been investigated.The present study demonstrated that the expression of SIRT6 was downregulated in osteosarcoma tissues and osteosarcoma cell lines when compared with adjacent tissues or osteoblastic cell lines. Kaplan-Meier analysis was performed to evaluate the prognostic significance of SIRT6. The overall survival of patients with higher expression of SIRT6 was significantly longer than patients with lower expression. Subsequently, MTT and invasion assays were performed to detect the biological functions of SIRT6 in osteosarcoma cells in vitro. The results revealed that overexpression of SIRT6 inhibited SAOS-2 and MG-63 cell proliferation and invasion. Knockdown of SIRT6 enhanced cell ability for the proliferation and invasion. A qChIP assay, luciferase reporter assay, reverse transcription-quantitative polymerase chain reaction and western blotting confirmed that $\mathrm{CDH} 2$ (N-cadherin) was a target of SIRT6. SIRT6 overexpression suppressed N-cadherin on the mRNA and protein levels. In addition, it was confirmed that the promotional effect of Si-SIRT6 on OS cell growth and invasion was suppressed by downregulating $\mathrm{N}$-cadherin. The present study suggested that SIRT6 may serve as a tumor suppressor during the development of osteosarcoma. In addition, $\mathrm{N}$-cadherin may be a promising therapeutic target for osteosarcoma.
\end{abstract}

Correspondence to: Professor Yong Ma, Department of Orthopedics and Traumatology, First Clinical Medical College, Nanjing University of Traditional Chinese Medicine, 138 Xianlin Road, Nanjing, Jiangsu 210023, P.R. China

E-mail: f4q1o1@163.com

Key words: SIRT6, proliferation, invasion, N-cadherin, osteosarcoma

\section{Introduction}

Osteosarcoma (OS) is a commonly and highly invasive primary malignant mesenchymal tumors in bone, which usually affecting the metaphysis of long bones of adolescents and young adults $(1,2)$. Over the past decades, although early diagnosis and effective treatment have been achieved, neoadjuvant and adjuvant chemotherapy coupled with limb-sparing surgery remains the most effective regimens. The 5-year survival rate of patients is still very poor due to recurrent or metastatic OS (3). Since the cause of OS is associated with abnormal genetic and epigenetic changes, the identification of potential effector molecules targeting key oncogenes are vital for OS treatment $(4,5)$. Several studies have investigated the genes associated with proliferation and metastasis in OS. Among them, $\mathrm{CDH} 2$, $\mathrm{N}$-cadherin has become a new research hotspot in gene therapy. Gain-of-function of $\mathrm{N}$-cadherin was considered as a universal hallmark of epithelial-mesenchymal transition (6,7). N-cadherin was reported to promote tumorigenesis during different cancer progression such as esophageal cancer (8), breast cancer (9), germ cell tumours (10), thyroid cancer (11). Although it is well known that interfering of $\mathrm{N}$-cadherin function may prove beneficial in multiple cancers, the exact regulation about $\mathrm{N}$-cadherin in OS remains largely unknown.

SIRT6 is a member of the class III histone deacetylase family $(12,13)$. During idiopathic pulmonary fibrosis, SIRT6 could inhibit epithelial to mesenchymal transition (14). SIRT6 was reported to function as a tumor suppressor or oncogene depend on the types of cancer, which indicated SIRT6 was at the crossroads of multiple pathways (15-17). Until now, there is no report about the function of SIRT6 in OS. In the present study, we revealed the exact role of SIRT6 in the regulation of OS cell proliferation and invasion in vitro. We also investigated the associated underlying mechanisms, focusing on the repression of $\mathrm{N}$-cadherin in OS cells.

\section{Materials and methods}

Cell culture and reagents. The human OS cell lines, SAOS-2, MG-63, U2OS and one osteoblastic cell line hFOB were purchased from the American Type Culture Collection (Manassas, VA, USA) and incubated in RPMI-1640 medium 
(GE Healthcare, Chicago, IL, USA) supplement with $10 \%$ fetal bovine serum (FBS; Thermo Fisher Scientific, Inc., Waltham, MA, USA). $100 \mathrm{U} / \mathrm{ml}$ penicillin and $100 \mu \mathrm{g} / \mathrm{ml}$ streptomycin was added to the medium. All cells were incubated in a humidified atmosphere at $37^{\circ} \mathrm{C}$ with $5 \% \mathrm{CO}_{2}$. Lipofectamine 2000 (Thermo Fisher Scientific, Inc.) was used for transfection, according to the manufacturer's instructions.

Ethics statement and tissues samples. The Medical Ethics Committee of Nanjing University of Traditional Chinese Medicine had approved this research, and written informed consent was obtained from all patients. Primary OS tissues samples were collected from 112 patients who were diagnosed with OS based on histopathological evaluation and underwent surgical treatment in our hospital from 2008 to 2013 (Table I). The clinical stage was classified according to the sixth edition of the TNM classification of the International Union Against Cancer (UICC). Prior anticancer treatment (radiotherapy or chemotherapy) were excluded, the biopsies were frozen in liquid nitrogen immediately and stored at $-80^{\circ} \mathrm{C}$ until use. All OS patients were studied in a follow-up for overall survival.

Lentiviral transduction. The relative lentivirus was purchased from Shanghai Genepharma, and transduced into the target cells with $8 \mu \mathrm{g} / \mathrm{ml}$ polybrene (Sigma-Aldrich; Merck KGaA, Darmstadt, Germany). $48 \mathrm{~h}$ after incubation, the cells were selected using puromycin $(2.0 \mu \mathrm{g} / \mathrm{ml}$; Sigma-Aldrich; Merck $\mathrm{KGaA}$ ). Following puromycin selection for $48 \mathrm{~h}$, the cells were harvest and used for RT-qPCR or western blot analysis.

$R N A$ extraction and $R T-q P C R$. Total RNA was extracted from the relative cells or tissues using TRIzol ${ }^{\circledR}$ reagent (Invitrogen; Thermo Fisher Scientific, Inc.) according to the manufacturer's instructions. $2 \mu \mathrm{g}$ RNA was transcribed into cDNA using iScript cDNA Synthesis kit (Bio-Rad Laboratories Inc., Hercules, CA, USA). RT-qPCR was performed on the Applied Biosystems 7500 Real-Time PCR System (Thermo Fisher Scientific, Inc.). The expression of GAPDH was used as a control. The primers for N-cadherin were forward, 5'-GTCAGCAGAAGT TGAAGAAATAGTG-3' and reverse, 5'-GCAAGTTGATTG GAGGGATG-3'. The primers for SIRT6 were forward, 5'-CTT TGTTACTTGTTTCTGTCCC-3' and reverse, 5'-GAC AACACAGCAAGTCAGAG-3'. The primers for GAPDH were forward, 5'-TGGGTGTGAACCATGAGAAGT-3' and reverse, 5'-TGAGTCCTTCCACGATACCAA-3'. Relative gene expression was calculated according to the $2^{-\Delta 4} \mathrm{Cq}$ method (18).

Western blott analysis. Proteins were extracted from cells using the lysis buffer (Beyotime Institute of Biotechnology, Nanjing, China) in the presence of a proteinase inhibitor cocktail (Complete Mini; Roche Diagnostics, Basel, Switzerland). After centrifugation at $15,000 \mathrm{~g}$ for $15 \mathrm{~min}$ at $4^{\circ} \mathrm{C}$, the supernatants were quantified using Bradford assay reagent (Thermo Fisher Scientific International Inc.) $30 \mu \mathrm{g}$ proteins in each group were separated on 10\% SDS-PAGE and then blotted onto a polyvinylidene difluoride (PVDF) membrane (GE Healthcare). After blocked with 5\% non-fat dried milk in TBST (Sigma-Aldrich; Merck KGaA) overnight at $4^{\circ} \mathrm{C}$. The membrane was incubated with specific primary antibodies at room temperature for $3 \mathrm{~h}$, respectively.
Anti- $\beta$-actin (cat no. 612656; 1:2,000; BD Biosciences, Franklin Lakes, NJ, USA). Anti-SIRT6 (ab 62739; 1:1,000; polyclonal rabbit); Anti-N-cadherin (ab18203; 1:1,000; polyclonal rabbit; both Abcam, Cambridge, MA, USA). HRP-linked goat anti-rabbit (cat no. 7074) or goat anti-mouse secondary antibodies (cat no. 7076), both from Cell Signaling Technology, Inc. were used for incubation for $1 \mathrm{~h}$ at $37^{\circ} \mathrm{C}$. The chemiluminescent horseradish peroxidase substrate (Merck KGaA, Darmstadt, Germany) was used to detect the signals according to the manufacturer's instructions.

MTT assay. Cell proliferation was performed using MTT assay (Dojindo, Kumamoto, Japan), according to the manufacturer's instructions. Briefly, the cells were plated in 96-well plates. $5 \times 10^{3}$ cells each well were harvested at $24,48,72$ and $96 \mathrm{~h} .10 \mu \mathrm{l} \mathrm{CCK}-8$ solution was added to each well with $100 \mu 1 \mathrm{RPMI}-1640$. The plates were further incubated at $37^{\circ} \mathrm{C}$ for $2 \mathrm{~h}$ and the absorbance at $490 \mathrm{~nm}$ was measured using a microplate reader (Infinite Pro 2000; Tecan GmbH, Grödig, Austria). All experiments were performed at least three times.

Cell invasion assay. To assess the role of SIRT6 in cell invasion, Transwell assay was performed. Transwell chambers were purchased from Merck KGaA (24-well; $8 \mu \mathrm{m})$. The filter of the upper chamber was coated with $50 \mu 1$ of diluted Matrigel (BD Biosciences), according to the manufacturer's instructions. The lower chambers were filled with $600 \mu 1$ of RPMI-1640 with $10 \%$ FBS. A total of $1 \times 10^{5}$ cells were suspended in $100 \mu 1$ serum free RPMI-1640 medium and added into each top chamber. After the cells were incubated for $10 \mathrm{~h}$ at $37^{\circ} \mathrm{C}$ in a $5 \% \mathrm{CO}_{2}$ chamber, the non-invaded cells were removed with a cotton swab.

The invasive cells on the lower surface of the membrane were were fixed with $4 \%$ paraformaldehyde for $25 \mathrm{~min}$ and then stained with $0.1 \%$ crystal violet for $10 \mathrm{~min}$. The invasive cells were counted under a microscope (Leica DM 5000 B; Leica Microsystems, Wetzlar, Germany) in five random selected fields. The experiments were repeated at least three times and results were expressed as the relative fold change over vector.

$q$ ChIP assay. qChIP was performed using kit from Upstate Biotechnology according to manufacturer's instructions, Sybr-Green (Molecular Probes, Eugene, OR, USA) was used as a marker for DNA amplification on the ABI 7500 system.

Luciferase reporter assay. Luciferase reporter assay was used to clarify whether $\mathrm{N}$-cadherin was a direct target of SIRT6. The promoter of $\mathrm{CDH} 2$ was cloned into the pRL-TK report vector (Thermo Fisher Scientific, Inc.). The relative cells were transfected with the wild-type or mutant type promoter of $\mathrm{CDH} 2$ and SIRT6 or Si-SIRT6, using Lipofectamine 2000, respectively. $48 \mathrm{~h}$ later, luciferase activity was determined using a dual luciferase reporter assay (Promega Corp., Madison, WI, USA) and LD400 luminometer (Beckman Coulter, Inc., Brea, CA, USA). Data was presented as the ratio of Renilla luciferase to that of the firefly luciferase. All experiments were repeated at least three times.

Statistical analysis. SPSS 18.0 software (SPSS, Inc., Chicago, IL, USA) was used to perform statistical analysis. Data was expressed as mean \pm standard deviation for at least three separate experiments. $\mathrm{P}<0.05$ was considered to indicate a statistically 
Table I. Clinicopathologic variables in 112 osteosarcoma cancer patients.

\begin{tabular}{|c|c|c|c|c|}
\hline \multirow[b]{2}{*}{ Variables } & \multirow[b]{2}{*}{$\begin{array}{c}\text { No. } \\
(n=112)\end{array}$} & \multicolumn{2}{|c|}{ SIRT6 expression } & \multirow[b]{2}{*}{ P-value } \\
\hline & & $\begin{array}{c}\text { Low } \\
(n=72)\end{array}$ & $\begin{array}{l}\text { High } \\
(n=40)\end{array}$ & \\
\hline Age (years) & & & & 0.352 \\
\hline$<40$ & 55 & 33 & 22 & \\
\hline$\geq 40$ & 57 & 39 & 18 & \\
\hline Sex & & & & 0.284 \\
\hline Male & 54 & 32 & 22 & \\
\hline Female & 58 & 40 & 18 & \\
\hline Histological grade & & & & 0.002 \\
\hline I-II & 48 & 23 & 25 & \\
\hline III & 64 & 49 & 15 & \\
\hline Enneking staging & & & & 0.032 \\
\hline I-II & 52 & 28 & 24 & \\
\hline III & 60 & 44 & 16 & \\
\hline Metastasis & & & & $<0.001$ \\
\hline Yes & 57 & 46 & 11 & \\
\hline No & 55 & 26 & 29 & \\
\hline
\end{tabular}

SIRT6, sirtuin-6.

significant difference. Student's t test and one-way analysis of variance (ANOVA) were used to analyze the difference. Cox regression (proportional hazards model) was used to perform multivariate analysis of prognostic factors. The log-rank test was used to determine the patient survival and the differences.

\section{Results}

SIRT6 was downregulated in the OS tissues and cell lines. In order to study the role of SIRT6 in OS, we first examined the expression of SIRT6 in OS tissues, as shown in Fig. 1A, SIRT6 was downregulated in the OS tissues than in the adjacent non-tumor soft tissues. Meanwhile, the expression of SIRT6 was also lower in the OS cell lines SAOS-2, MG-63 and U2OS compared with the osteoblastic cell line hFOB. We then chose SAOS-2 and MG-63 to perform functional analysis of SIRT6. As collected, the follow-up information was available for all patients, Kaplan-Meier curve was analyzed to show that the higher expression of SIRT6 was statistically correlated with favorable overall survival rates in OS (Fig. 1C), $(\mathrm{P}=0,0381)$. With confidence intervals (1.04 4.36) and Hazard Ratio=2.13.

SIRT6 inhibited the proliferation of human OS cells. To confirm whether SIRT6 has any effect on the proliferation of OS cells. We established SAOS-2 and MG-63 overexpression SIRT6 cell lines using lentivirus carrying SIRT6 construct. As shown in Fig. 2A, the expression of SIRT6 was significantly increased after transfection with SIRT6 construct compared to the control group (Fig. 2A). Furthermore, we used two specific siRNA targeting SIRT6 to knockdown the expression of SIRT6. RT-qPCR assay was performed, as expected, Si-SIRT6
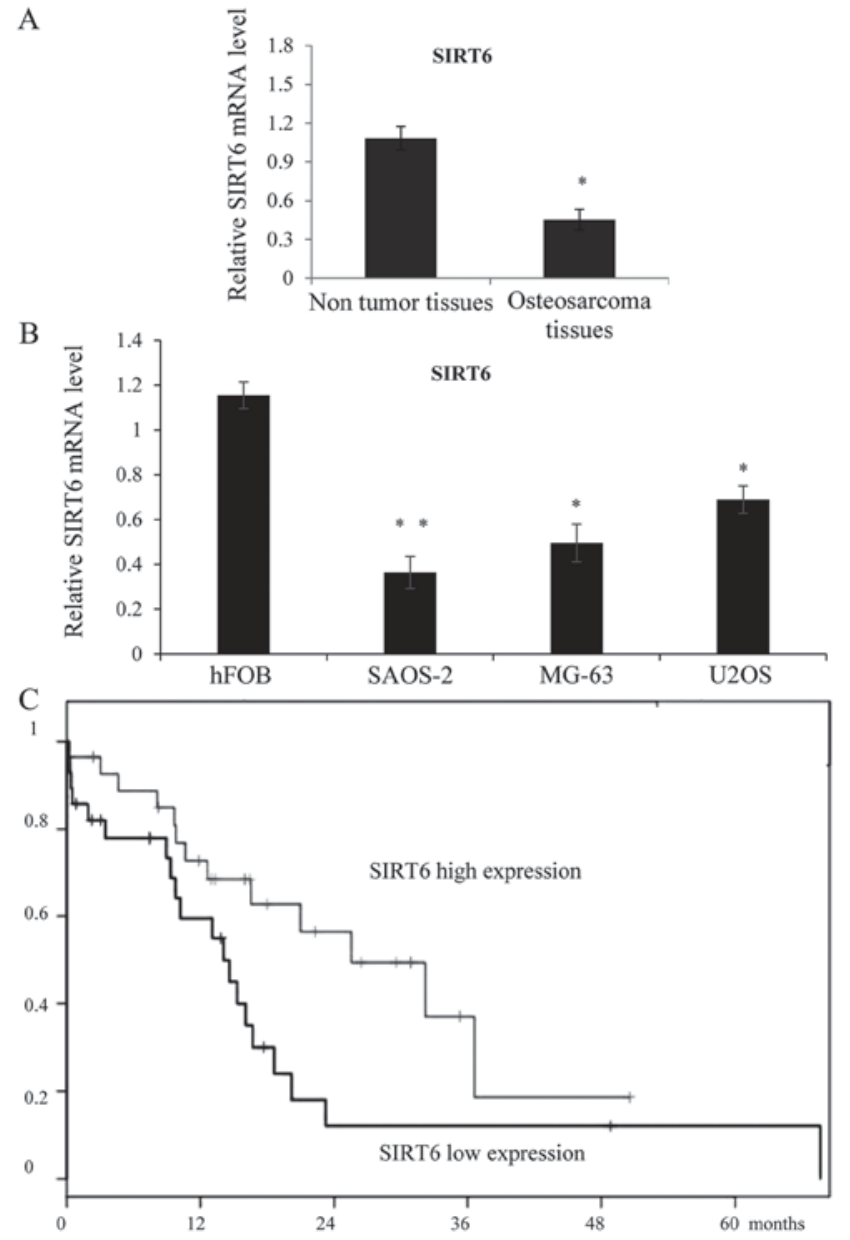

Figure 1. SIRT6 was downregulated in the osteosarcoma tissues and cell lines. (A) RT-qPCR was performed to evaluate mRNA expression of SIRT6 in osteosarcoma tissues and their matched non-tumor tissues. The expression of SIRT6 was normalized to GAPDH levels. "P $<0.05$ vs. non-tumor tissues. (B) RT-qPCR was performed to examine the relative expression of SIRT6 in SAOS-2, MG-63, U2OS cells and the osteoblastic cell line hFOB. ${ }^{~} \mathrm{P}<0.05$, ${ }^{* *} \mathrm{P}<0.01$ vs. hFOB. (C) Increased expression of SIRT6 was correlated with better overall survival in osteosarcoma patients. The median SIRT6 level in the tumor samples was chosen as the cut-off point. Kaplan-Meier blot was drawn and Log-rank tests was used to show the differences. ${ }^{*} \mathrm{P}<0.05$ vs. lower SIRT6 expression. SIRT6, sirtuin-6; RT-qPCR, reverse transcription-quantitative polymerase chain reaction.

could remarkably reduce the level of SIRT6 (Fig. 2B). Overexpression of SIRT6 decreased the proliferation rate of SAOS-2 and MG-63 cells, on the other hand, knockdown of SIRT6 dramatically enhanced cell proliferation (Fig. 2C).

SIRT6 inhibited the invasion of human OS cells. In order to investigate the function of SIRT6 on the invasion of OS cells, Transwell invasion assay was performed in the above two human OS cells. As shown in Fig. 3A, the invasion capacity of SAOS-2 transfected with SIRT6 was significantly reduced, as compared with the control groups. Moreover, SIRT6 overexpression also suppressed the invasive capacity of MG-63 cells, when compared to the control groups (Fig. 3B). By contrast, the knockdown of SIRT6 had significantly promotion effect on the invasion of the SAOS-2 cells and MG-63 cells (Fig. 3C and D).

Identification of $N$-cadherin as a direct target of SIRT6. We next investigated the molecular mechanism of SIRT6 

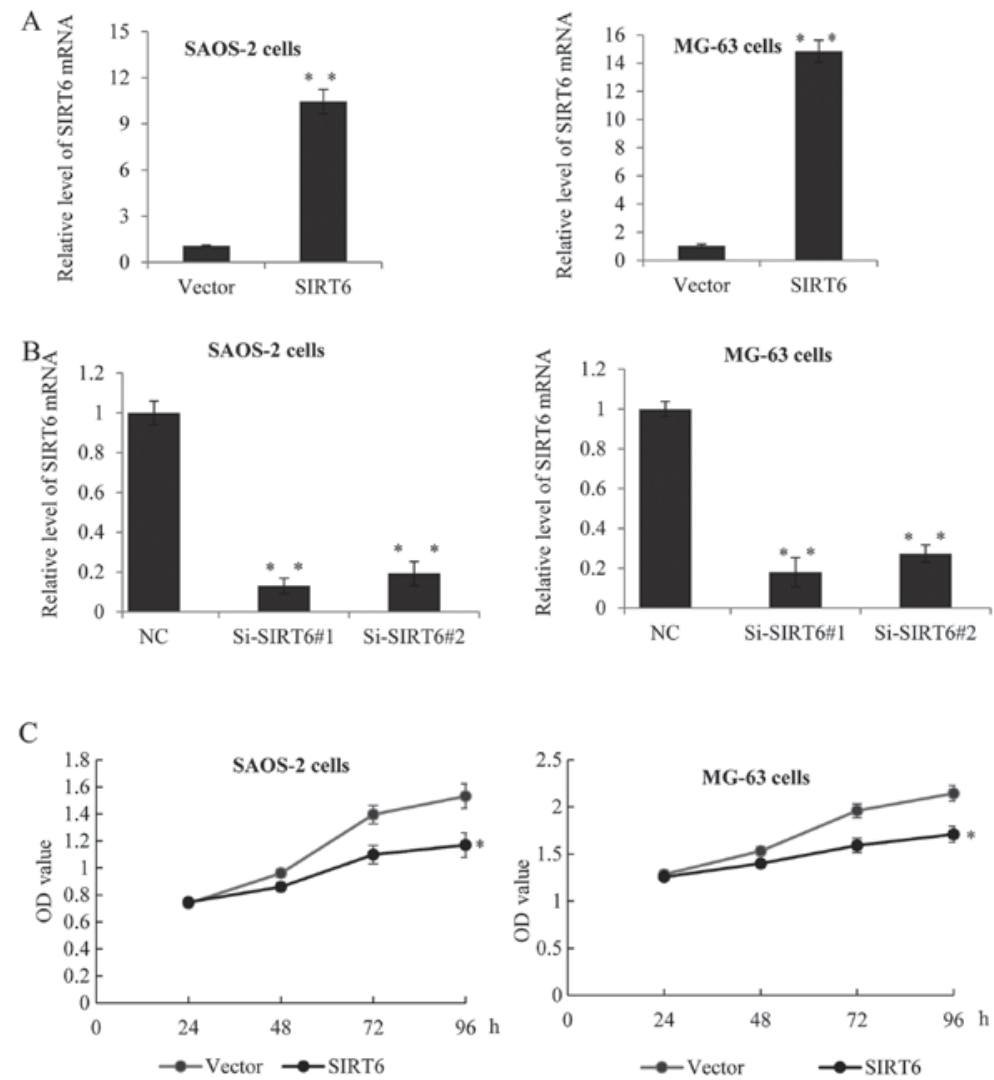

Figure 2. SIRT6 inhibited the proliferation of human osteosarcoma cells. (A) RT-qPCR was used to analyze SIRT6 expression in SAOS-2 and MG-63 cells transfected with SIRT6 construct or vector, or transfected with control siRNA or (B) Si-SIRT6\#1, or Si-SIRT6\#2. GAPDH was used as an internal control. Data was presented as means $\pm \mathrm{SD}$, of three independent experiments, ${ }^{* *} \mathrm{P}<0.01$ vs. vector or NC. (C) MTT assay was performed in SAOS-2 cells or MG-63 cells transfected with control or SIRT6 construct, or transfected with control siRNA or Si-SIRT6\#1, or Si-SIRT6\#2. The absorbance at 490 nm was measured at 0, 24, 48, 72 and $96 \mathrm{~h}$, of three independent experiments, "P<0.05 vs. vector or NC. SIRT6, sirtuin-6; RT-qPCR, reverse transcription-quantitative polymerase chain reaction.
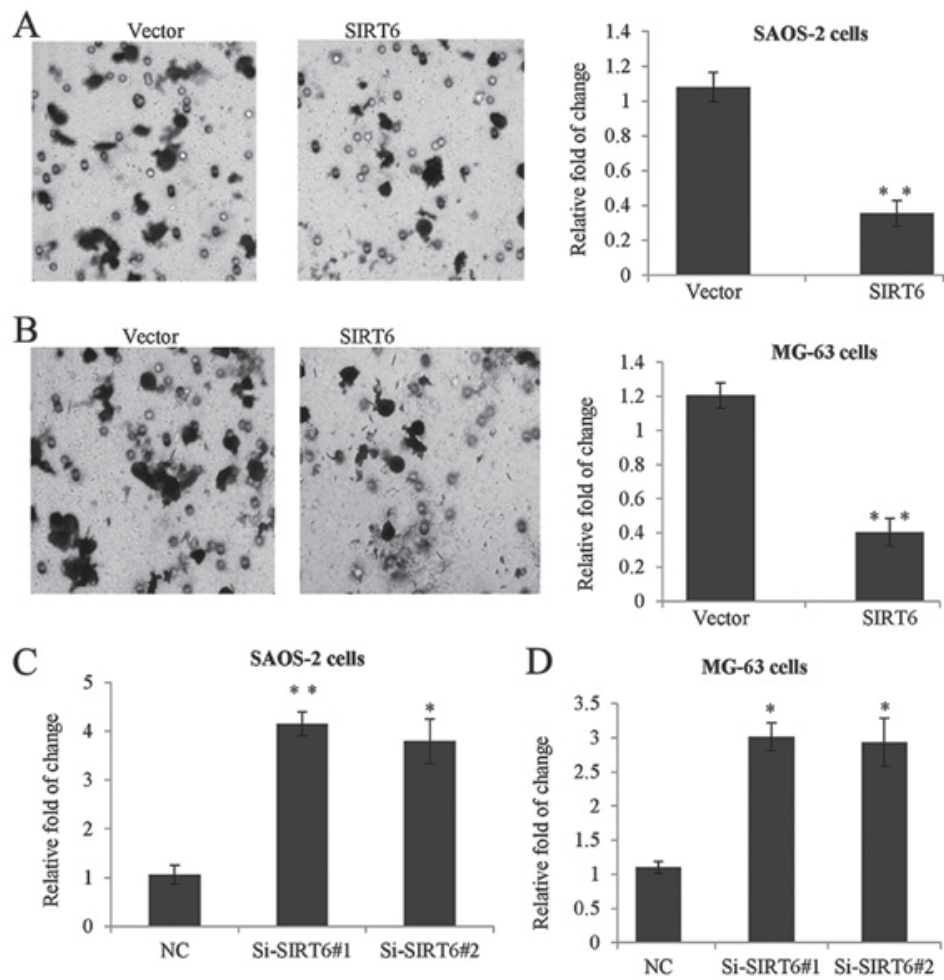

Figure 3. SIRT6 inhibited the invasion of human osteosarcoma cells. (A) Transwell assay was performed in SAOS-2 cells or (B) MG-63 cells to determine the invasive capacity of cells transfected with SIRT6 construct or vector, respectively. Representative images were shown (magnification, x20), and data were presented as the mean \pm standard deviation, of three independent experiments, ${ }^{* *} \mathrm{P}<0.01$ vs. vector. (C) SAOS-2 cells transfected with control siRNA or Si-SIRT6\#1, or Si-SIRT6\#2 or (D) MG-63 cells. The invasion potential was determined using a Transwell assay. "P<0.05, ${ }^{* *} \mathrm{P}<0.01$ vs. NC. SIRT6, sirtuin-6. 
A

SAOS-2 cells

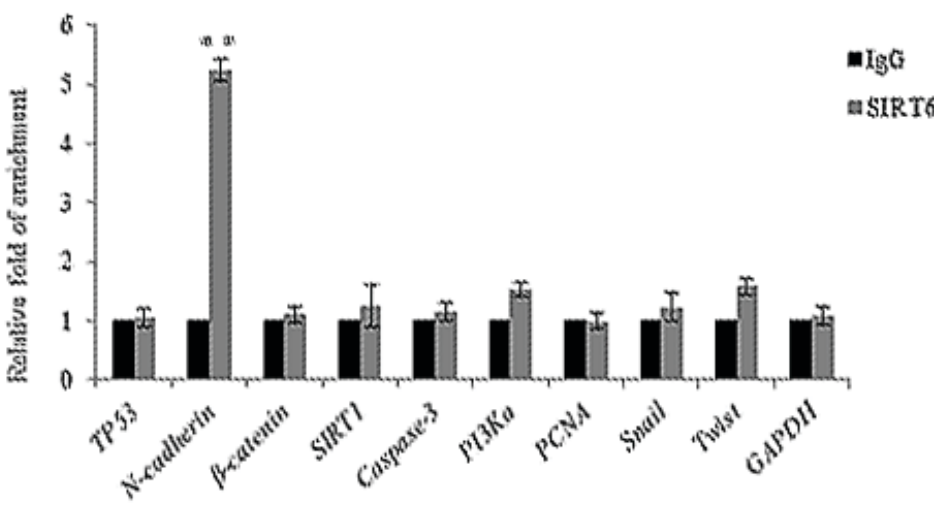

B

MGG 63 cells
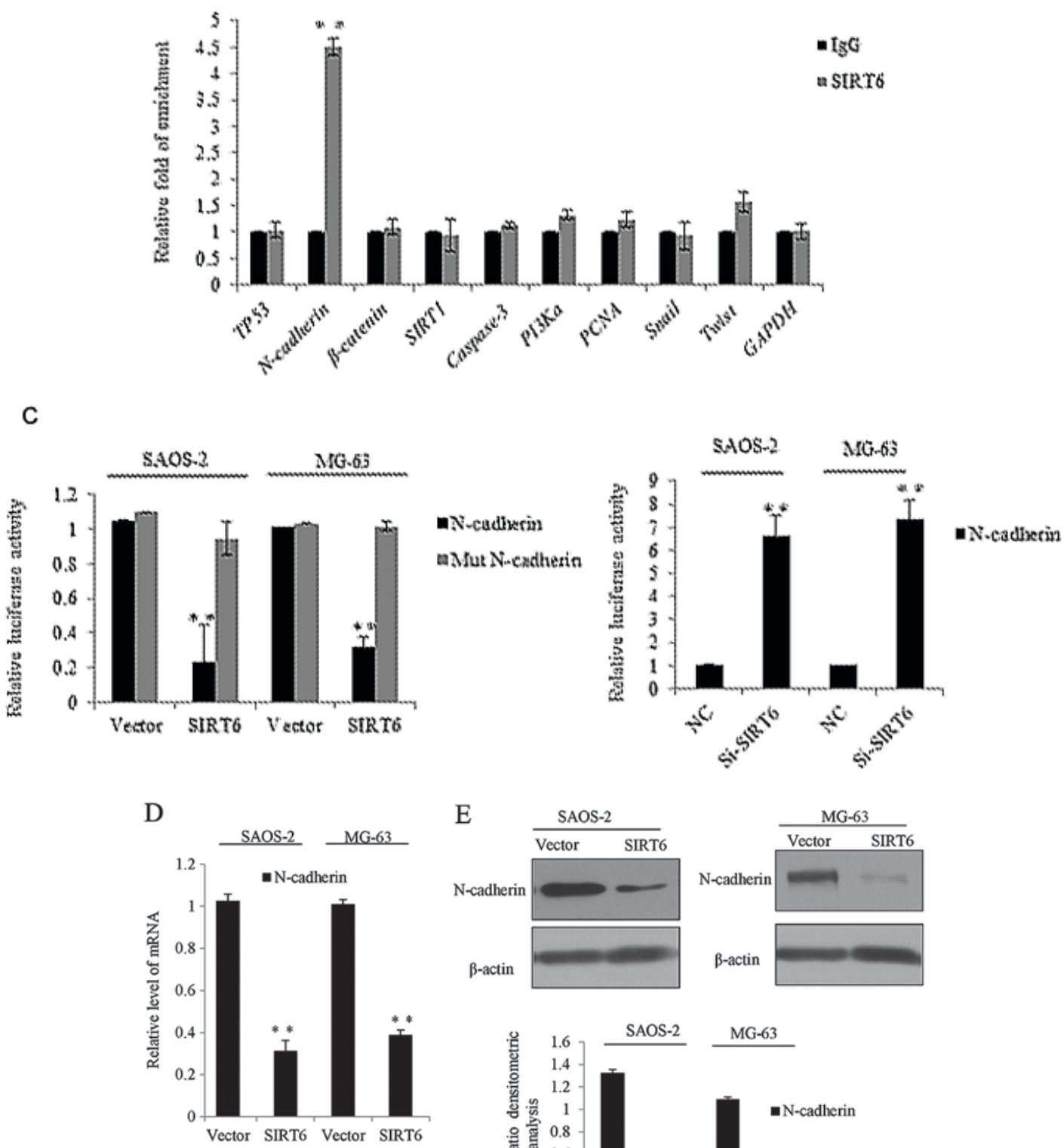

$\mathrm{E}$
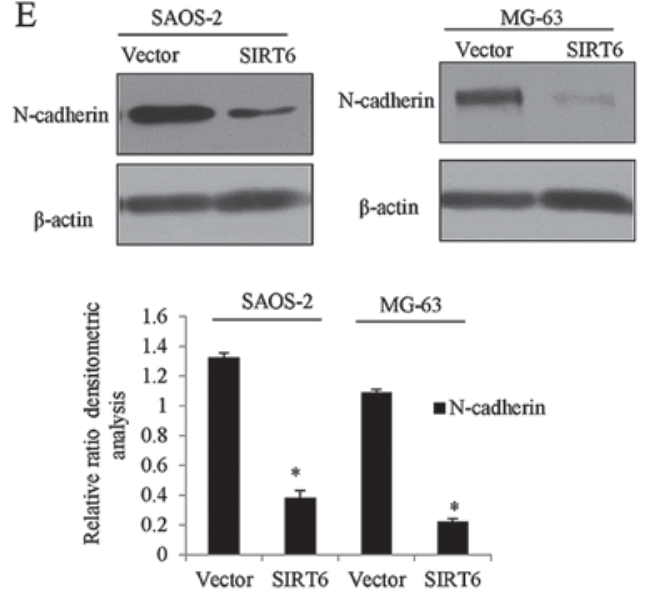

Figure 4. Identification of N-cadherin as a direct target of SIRT6. (A) qChIP experiment was performed in SAOS-2 cells or (B) MG-63 cells with indicated SIRT6 or normal IgG as a control. ${ }^{* *} \mathrm{P}<0.01$ vs. IgG. (C) Luciferase reporter assay was performed in SAOS-2 cells or MG-63 cells co-transfection with SIRT6 plasmid and WT CDH2 or mutant CDH2 promoter or Si-SIRT6 and WT CDH2 promoter. Luciferase activities were measured and normalized to the Renilla. ${ }^{* *} \mathrm{P}<0.01$ vs. vector or NC. (D) N-cadherin expression levels were evaluated by RT-qPCR analysis or western blot (E) in SAOS-2 cells or MG-63 cells transfected vector or SIRT6. ${ }^{* *} \mathrm{P}<0.01$ vs. vector. SIRT6, sirtuin-6; WT, wild-type; CDH2, N-cadherin. 

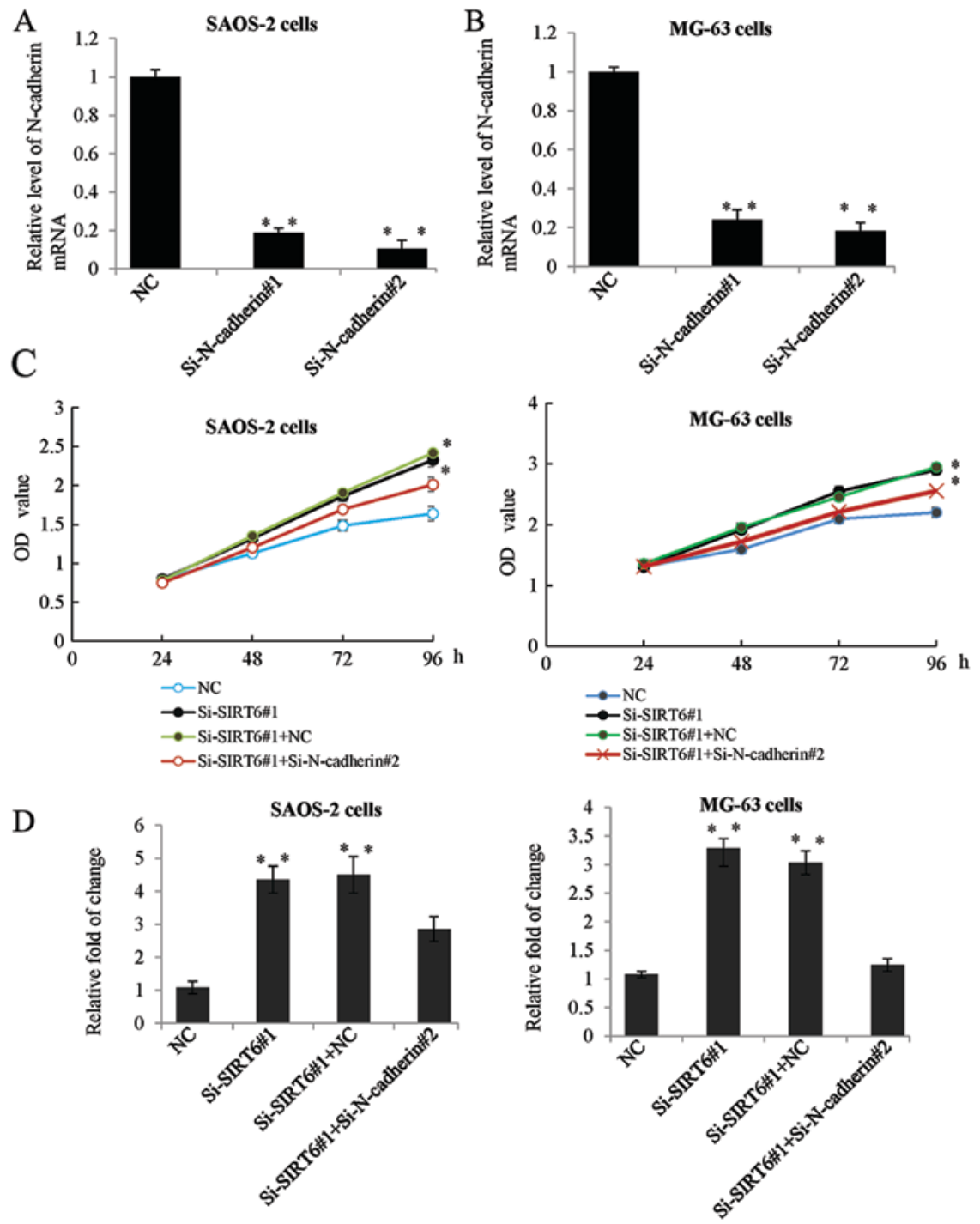

Figure 5. N-cadherin was involved in SIRT6 mediated proliferation and invasion of OS cells. (A) RT-qPCR was performed to determine the mRNA level of $\mathrm{N}$-cadherin in SAOS-2 cells or (B) MG-63 cells transfected with control siRNA or Si-N-cadherin\#1, or Si-N-cadherin\#2. GAPDH was used as an internal reference. ${ }^{* *} \mathrm{P}<0.01$ vs. NC. (C) The MTT assay (D) The Transwell assay was carried out in SAOS-2 cells or MG-63 cells transfected with control siRNA or Si-SIRT6\#1 or co-transfected with Si-SIRT6\#1 and control siRNA or Si-N-cadherin. "P<0.05 vs. NC. SIRT6, sirtuin-6; OS, osteosarcoma; RT-qPCR, reverse transcription-quantitative polymerase chain reaction.

mediated biological functions. We examined its potential targets by searching quantitative ChIP assays in SAOS-2 cells and MG-63 cells. Several key genes in different pathways were chosen, as shown in Fig. 4A and B, on the promoter of $\mathrm{CDH} 2$ (N-cadherin), there was obviously binding enrichment of SIRT6. To further support the argument, luciferase reporter activity assay was carried out, the SAOS-2 cells and MG-63 cells transfected with SIRT6 significantly repressed the luciferase activity of the wild-type $\mathrm{CDH} 2$ promoter, whereas the mutation of the promoter abolished this repression, oppositely, the knockdown of SIRT6 resulted in the enhanced $\mathrm{CDH} 2$ reporter activity (Fig. 4C). Consistently, we detected the mRNA and protein level of N-cadherin in the SAOS-2 cells and MG-63 cells transfected with the SIRT6 overexpression plasmid. As shown in Fig. 4D and E, overexpression of SIRT6 inhibited the level of $\mathrm{N}$-cadherin in the OS cells as compared to the vector groups. Together, the above data demonstrated that N-cadherin was a direct target of SIRT6, and SIRT6 reduced $\mathrm{N}$-cadherin expression.
$N$-cadherin was involved in SIRT6 mediated proliferation and invasion of $O S$ cells. To further clarify whether the inhibition of N-cadherin was as a downstream effector in SIRT6 mediated proliferation and invasion of OS cells. Firstly, we determined the mRNA expression of $\mathrm{N}$-cadherin in the SAOS-2 and MG-63 cells transfected with siRNA targeting $\mathrm{N}$-cadherin. As shown in Fig. 5A and B, transfection with $\mathrm{Si}-\mathrm{N}$-cadherin significantly inhibited the expression level of $\mathrm{N}$-cadherin. Furthermore, we transfected SAOS-2 and MG-63 cells with SIRT6 siRNA or co-transfected with N-cadherin siRNA. MTT assay and Transwell assay was performed respectively. While transfection with $\mathrm{Si}-\mathrm{N}$-cadherin reduced the promotion effect of Si-SIRT6 on OS cells proliferation and invasion (Fig. 5C and D).

\section{Discussion}

SIRT6 was one of the NAD+-dependent deacetylase sirtuin family. Sirtuins had seven members in mammals and were 
widely expressed in different tissues. Among them, SIRT6 was a broadly expressed, predominantly nuclear protein (19). As reported, the major function of SIRT6 is to maintenance genomic integrity (20). SIRT6 was identified as a tumor suppressor and regulated aerobic glycolysis in cancer cells (13), furthermore, SIRT6 was reported to cooperate with tumor suppressor p53 to regulate gluconeogenesis (21). Marquardt et al (22) showed SIRT6 was significantly down-regulated in hepatocellular carcinoma and the dysregulated genes by loss of SIRT6 displayed oncogenic effects in HCC. Feng et al (23) reported in glioma, the expression of SIRT6 was significantly lower in glioblastoma multiform tissues and SIRT6 suppressed glioma cell growth via induction of apoptosis by inhibition of JAK2/STAT3 activation. However, the correlation between SIRT6 and OS has not been investigated.

Significant difference of SIRT6 expression was found in OS tissues and adjacent non-cancerous tissues, as shown in Table I. A higher expression of SIRT6 was associated with a higher histological grade and higher enneking staging. Higher expression of SIRT6 indicative of better overall survival times, at 24 months, the difference was major, however, at 36 months, since most patients died, the difference has vanished, which indicated SIRT6 might be involved in the development of OS. As known, during tumor progression, there were two essential steps as uncontrolled cell proliferation and aggressive cell metastasis, so in our study, we focus on whether SIRT6 has a function in the OS tumor growth and metastasis. We also performed apoptosis analysis in the preliminary experiments, but there is no obvious difference between the SIRT6 group and the vector group, data was not shown. Although Kok et al (24) reported SIRT6 modulated hypoxia-induced apoptosis in osteoblasts, but in OS, there is no exact evidence to confirm that. Furthermore, as Sugatani et al (25) reported, SIRT6 deficiency culminated in low-turnover osteopenia. As Zhang et al (26) reported, SIRT6 deficiency could cause senile osteoporosis. The above observation together with us revealed multiple function of SIRT6 and OS, osteoporosis and osteopenia.

The results of the present study demonstrated that SIRT6 inhibited the proliferation and invasion of the SAOS-2 and MG-63 OS cell lines, further investigations such as qChIP and luciferase reporter assay demonstrated that $\mathrm{N}$-cadherin was a direct target inhibited by SIRT6. The expression of $\mathrm{N}$-cadherin was opposite with the SIRT6 status from mRNA and protein level. Therefore we made the hypothesis that $\mathrm{N}$-cadherin might be involved in the regulation by SIRT6 in the progression of OS cells. The evidences were as follows: By reduced the expression of $\mathrm{N}$-cadherin under SIRT6 knockdown, the promotion phenotypes of Si-SIRT6 could be almostly reduced. Although further investigation was required for characterization of other targets involved in SIRT6 inhibiting OS. The molecular mechanisms underlying SIRT6 as a tumor suppressor gene in OS may prove to be a promising gene therapeutic agent.

\section{Competing interests}

The authors declare that they have no conflicts of interest about this article.

\section{References}

1. Mirabello L, Troisi RJ and Savage SA: Osteosarcoma incidence and survival rates from 1973 to 2004: Data from the surveillance, epidemiology and end results program. Cancer 115: 1531-1543, 2009.

2. Bielack S, Carrle D and Casali PG; ESMO Guidelines Working Group: Osteosarcoma: ESMO clinical recommendations for diagnosis, treatment and follow-up. Ann Oncol 20 (Suppl 4): S137-S139, 2009.

3. Bielack SS, Kempf-Bielack B, Delling G, Exner GU, Flege S, Helmke K, Kotz R, Salzer-Kuntschik M, Werner M, Winkelmann W, et al: Prognostic factors in high-grade osteosarcoma of the extremities or trunk: An analysis of 1,702 patients treated on neoadjuvant cooperative osteosarcoma study group protocols. J Clin Oncol 20: 776-790, 2002.

4. Marina N, Gebhardt M, Teot L and Gorlick R: Biology and therapeutic advances for pediatric osteosarcoma. Oncologist 9: 422-441, 2004

5. Kansara M, Teng MW, Smyth MJ and Thomas DM: Translational biology of osteosarcoma. Nat Rev Cancer 14: 722-735, 2014.

6. Nieman MT, Prudoff RS, Johnson KR and Wheelock MJ: $\mathrm{N}$-cadherin promotes motility in human breast cancer cells regardless of their E-cadherin expression. J Cell Biol 147: 631-644, 1999.

7. Islam S, Carey TE, Wolf GT, Wheelock MJ and Johnson KR: Expression of $\mathrm{N}$-cadherin by human squamous carcinoma cells induces a scattered fibroblastic phenotype with disrupted cell-cell adhesion. J Cell Biol 135: 1643-1654, 1996.

8. Nishitani S, Noma K, Ohara T, Tomono Y, Watanabe S, Tazawa H, Shirakawa Y and Fujiwara T: Iron depletion-induced downregulation of $\mathrm{N}$-cadherin expression inhibits invasive malignant phenotypes in human esophageal cancer. Int J Oncol 49: 1351-1359, 2016.

9. Park KS, Dubon MJ and Gumbiner BM: N-cadherin mediates the migration of MCF-10A cells undergoing bone morphogenetic protein 4-mediated epithelial mesenchymal transition. Tumour Biol 36: 3549-3556, 2015.

10. Bremmer F, Schallenberg S, Jarry H, Küffer S, Kaulfuss S, Burfeind P, Strauß A, Thelen P, Radzun HJ, Ströbel P, et al: Role of N-cadherin in proliferation, migration, and invasion of germ cell tumours. Oncotarget 6: 33426-33437, 2015.

11. Da C, Wu K, Yue C, Bai P, Wang R, Wang G, Zhao M, Lv Y and Hou P: N-cadherin promotes thyroid tumorigenesis through modulating major signaling pathways. Oncotarget 8: 8131-8142, 2017.

12. Michishita E, McCord RA, Berber E, Kioi M, Padilla-Nash H, Damian M, Cheung P, Kusumoto R, Kawahara TL, Barrett JC, et al: SIRT6 is a histone H3 lysine 9 deacetylase that modulates telomeric chromatin. Nature 452: 492-496, 2008.

13. Sebastián C, Zwaans BM, Silberman DM, Gymrek M, Goren A Zhong L, Ram O, Truelove J, Guimaraes AR, Toiber D, et al: The histone deacetylase SIRT6 is a tumor suppressor that controls cancer metabolism. Cell 151: 1185-1199, 2012.

14. Tian K, Chen P, Liu Z, Si S, Zhang Q, Mou Y, Han L, Wang Q and Zhou X: Sirtuin 6 inhibits epithelial to mesenchymal transition during idiopathic pulmonary fibrosis via inactivating TGF- $\beta 1 / \mathrm{Smad} 3$ signaling. Oncotarget 8 : 61011-61024, 2017.

15. Bai L, Lin G, Sun L, Liu Y, Huang X, Cao C, Guo Y and Xie C: Upregulation of SIRT6 predicts poor prognosis and promotes metastasis of non-small cell lung cancer via the ERK1/2/MMP9 pathway. Oncotarget 7: 40377-40386, 2016.

16. Kugel S, Sebastián C, Fitamant J, Ross KN, Saha SK, Jain E, Gladden A, Arora KS, Kato Y, Rivera MN, et al: SIRT6 suppresses pancreatic cancer through control of Lin28b. Cell 165: 1401-1415, 2016.

17. Elhanati S, Ben-Hamo R, Kanfi Y, Varvak A, Glazz R, Lerrer B, Efroni S and Cohen HY: Reciprocal regulation between SIRT6 and miR-122 controls liver metabolism and predicts hepatocarcinoma prognosis. Cell Rep 14: 234-242, 2016

18. Livak KJ and Schmittgen TD: Analysis of relative gene expression data using real-time quantitative PCR and the 2(-Delta Delta C(T)) method. Methods 25: 402-408, 2001.

19. Liszt G, Ford E, Kurtev M and Guarente L: Mouse Sir2 homolog SIRT6 is a nuclear ADP-ribosyltransferase. J Biol Chem 280: 21313-21320, 2005. 
20. Mostoslavsky R, Chua KF, Lombard DB, Pang WW, Fischer MR Gellon L, Liu P, Mostoslavsky G, Franco S, Murphy MM, et al: Genomic instability and aging-like phenotype in the absence of mammalian SIRT6. Cell 124: 315-329, 2006.

21. Zhang P, Tu B, Wang H, Cao Z, Tang M, Zhang C, Gu B, Li Z, Wang L, Yang Y, et al: Tumor suppressor p53 cooperates with SIRT6 to regulate gluconeogenesis by promoting FoxO1 nuclear exclusion. Proc Natl Acad Sci USA 111: 10684-10689, 2014.

22. Marquardt JU, Fischer K, Baus K, Kashyap A, Ma S, Krupp M, Linke M, Teufel A, Zechner U, Strand D, et al: Sirtuin-6-dependent genetic and epigenetic alterations are associated with poor clinical outcome in hepatocellular carcinoma patients. Hepatology 58: 1054-1064, 2013.

23. Feng J, Yan PF, Zhao HY, Zhang FC, Zhao WH and Feng M: SIRT6 suppresses glioma cell growth via induction of apoptosis, inhibition of oxidative stress and suppression of JAK2/STAT3 signaling pathway activation. Oncol Rep 35: 1395-1402, 2016.
24. Kok SH, Hou KL, Hong CY, Chao LH, Hsiang-Hua Lai E, Wang HW, Yang H, Shun CT, Wang JS and Lin SK: Sirtuin 6 modulates hypoxia-induced apoptosis in osteoblasts via inhibition of glycolysis: Implication for pathogenesis of periapical lesions. J Endod 41: 1631-1637, 2015.

25. Sugatani T, Agapova O, Malluche HH and Hruska KA: SIRT6 deficiency culminates in low-turnover osteopenia. Bone 81: 168-177, 2015.

26. Zhang DM, Cui DX, Xu RS, Zhou YC, Zheng LW, Liu P and Zhou XD: Phenotypic research on senile osteoporosis caused by SIRT6 deficiency. Int J Oral Sci 8: 84-92, 2016. 